\title{
Improved Dynamic PPI Network Construction and Application of Data Mining in Computer Artificial Intelligence Systems
}

\author{
Ruipeng Tang $\mathbb{D}$ \\ School of Computer and Electronic Information, Guangdong Institute of Petrochemical Technology, Maoming 525000, China \\ Correspondence should be addressed to Ruipeng Tang; tang_ruipeng@outlook.com
}

Received 26 November 2021; Revised 11 January 2022; Accepted 12 January 2022; Published 28 February 2022

Academic Editor: M Pallikonda Rajasekaran

Copyright ( 2022 Ruipeng Tang. This is an open access article distributed under the Creative Commons Attribution License, which permits unrestricted use, distribution, and reproduction in any medium, provided the original work is properly cited.

\begin{abstract}
The protein interaction network is a network composed of the interactions between all proteins in living organisms. The use of computational methods to prove the functional modules in the PPI network is an important research topic in bioinformatics. With the development of technologies for detecting protein interactions, the available data on interactions has become more and more extensive, and the PPI networks created have become more and more extensive. At the same time, with the deepening of research, people have found that the real PPI network is constantly changing with time and conditions and this change is closely related to the creation and development of life activities. Therefore, it is also very important and necessary to understand and study the functional modules of the PPI dynamic network. At the same time, it focuses on analyzing the basic attributes of artificial intelligence and computer technology and analyzing the goals that can be achieved by using artificial intelligence in computer technology. The effects and directions that can be further explored can be extracted from a large amount of unrealistic, incomplete, fuzzy, and random actual data. These data are hidden from people who do not know them before, but this is a potentially useful process for obtaining information and knowledge. Applying data mining technology to diagnose computer network problems can improve reliability, stability, flexibility, troubleshooting speed, and proper network management, automatically diagnose, predict, and maintain network failures, and ensure that the network has high-quality services and reliability. Drive AI applications in computer science.
\end{abstract}

\section{Introduction}

The information age is the main symbol of the 21st century society. Social progress and economic development are closely related to information, and computer networks are an important means of processing and applying information. Computer networks are different from conventional data transmission networks [1] and are the product of a combination of computer technology, communication technology, multimedia technology [2], optoelectronic technology, and other high-tech technologies. The information processed by the computer not only is limited to simple calculation tasks but also includes various files, forms, languages, audio and video files, graphics, and image task processing [3]. Therefore, the computer network has invaded all areas of society, and its scale is getting bigger and bigger [4].
Since the birth of computer networks, their reliability and availability have always been people's concerns, especially in the military, finance, and government, especially in the fields of science and technology [5]. With the rapid development and widespread use of computer networks, the scale of the network continues to expand, the network has become more and more complex and heterogeneous [6], and various new network applications have gradually appeared, such as real-time network video conferencing and modern distance education, and remote medical technologies such as those developed in [7], and the requirements for network reliability and availability have also increased. In modern society, computer science has become an important tool in production and life [8]. With the in-depth application of computer technology, traditional computer technology has gradually become more and more difficult to meet people's needs for intelligence and cutting-edge technology [9]. In 
this case, artificial intelligence begins to play a role [10], which has become an important direction for the development of information technology. Artificial intelligence technologies that are currently being explored in greater depth include expert systems, machine learning, and neural networks [11]. The application of these new technologies in computer technology, on the one hand, can meet the needs of information system users to a certain extent; on the other hand, it will also contribute to the development of informatics [12-15].

\section{Related Work}

In [16], it was proposed that, with the decoding of the whole genome sequence of a large number of living organisms and the development of functional genomics, protein is the functional unit in the cell and biologists are using more proteins and more complete biological data to analyze proteins. When studying omics, protein is also an irreplaceable basic component of cell and tissue structure. It is also the executor of life and the embodiment of biological functions. The performance of life activities is usually completed through the interaction of several proteins [17]. Therefore, studying the interaction between proteins is the basis for understanding the organization, process, and function of cells. It is known from [18] that the discovery of PPI network function modules is a process in which clustering technology is used to extract similar functions from PPI network data. We all know that because the discovery of functional modules can help people predict unknown functions and understand various biological processes, they can provide an important theoretical basis for studying the mechanism of diseases and finding new drug targets [19-21]. Therefore, in the past, data mining and machine tools have been used for ten years, and the rapid development of learning-based computer methods has also become the center of bioinformatics research.

Literature [22] suggests that the computer-aided design of equipment should be abbreviated as CAFD. The CAFD system is the core of international manufacturing research and development, using information technology to closely integrate the production links of the CAFD system company. Therefore, parameterization and rapid equipment design have become the focus of research in the field of CAFD. Particularly, in the transition from product development to mass production, rapid equipment manufacturing is essential to shorten the design cycle and prepare the equipment for production. Literature [23] shows that, with the current development of mechanical engineering, this is possible. As we all know, about $75 \%$ of companies are engaged in small-scale and mass production [23]. If a set of fixtures are developed for each production process of these products, this will seriously affect the company's cost and preproduction cycle. In order to solve this problem, we need to quickly assemble a set of fixtures suitable for processing different parts that need to be processed. After the parts are processed, the components of the equipment are removed for the next use, so the cycle is repeated. An expert system method is proposed to introduce artificial intelligence technology into the field of automatic diagnosis of communication network faults [24]. Mainframes with integrated circuits have become the mainstream of this century, and computing power and logic analysis capabilities have been significantly improved. The combination with network communication technology provides technical preparations for the birth of artificial intelligence [25]. However, the conventional network management technology cannot meet the requirements for the function, efficiency, and cost of network management [26].

\section{Improved Dynamic PPI Network Construction and Data Mining}

\subsection{Improved Dynamic PPI Network Construction}

3.1.1. PPI Function Module Detection and Dynamic PPI Network Overview. To establish a dynamic PPI network, this method basically evaluates the activity of a protein based on the protein expression level at a given time point. The proteins in the PPI dynamic network are composed of active proteins and their constant interactions. During this process, the activity threshold of each protein is determined by the following formula:

$$
\text { Active_Th }(p)=\mu(p)+3 \sigma(p)(1-F(p)) .
$$

$F(P)$ is defined as shown in the following formula:

$$
F(p)=\frac{1}{1+\sigma^{2}(p)} \text {. }
$$

Coexpression between proteins can be measured by normalization and correlation. The normalization method reflects the absolute level of expression between proteins, and the correlation reflects the relative level of expression between proteins [7]. The normalized value between proteins and its specific expression is shown in the following formula:

$$
D_{i}(x, y)=\frac{\left|G e_{i}(x)-G e(y)\right|}{G e_{i}(x)+G e_{i}(y)} .
$$

Table 1 shows the comparison of network topology attributes obtained by using three different methods to construct a dynamic PPI network for the same static PPI network data. Therefore, the density of the dynamic PPI network created by this method is low.

(1) Node Clustering Coefficient. Assuming that the number of adjacent nodes of node $p$ is equal to $d_{\mathrm{p}}$ and the number of connecting edges between these adjacent nodes is $n_{\mathrm{p}}$, the aggregation factor $\psi$ of node $p$ is as shown in the following formula:

$$
\psi_{p}=\frac{2 n_{p}}{d_{p} \cdot\left(d_{p}-1\right)} .
$$

The clustering factor of a node describes the closeness of connections between nodes adjacent to the node. The higher the value, the more important the position of the node in its 
TABle 1: Comparison of dynamic PPI network construction methods.

\begin{tabular}{lccc}
\hline Build a network approach & Average number of nodes & Average number of sides & Average density \\
\hline TC-PINs & 3204 & 12275 & 0.0024 \\
3-sigma & 637 & 934 & 0.0045 \\
Pearson's expression related & 3780 & 11368 & 0.0015 \\
\hline
\end{tabular}

field, and the more likely the opportunity to become the starting node of the cluster.

(2) Edge Cluster Coefficient. For the edge $E(x, y), \operatorname{Neigh}(x)$ and Neigh $(y)$ represent the data of neighbors $x$ and $y$, respectively, and the edge clustering factor is given in the following formula:

$$
\operatorname{ECC}(x, y)=\frac{|\operatorname{Neigh}(x) \cap \operatorname{Neigh}(y)|}{\min \{|\operatorname{Neigh}(x)|,|\operatorname{Neigh}(y)|\}} .
$$

The marginal aggregation coefficient describes the affinity between proteins.

(3) Pearson's Correlation Coefficient. Pearson's correlation coefficient can measure the degree of coexpression between proteins, and Pearson's correlation coefficient between proteins is usually used.

Calculate gene expression data and display specific definitions in the following formula:

$\operatorname{PCC}(x, y)=\frac{1}{n-1} \sum_{i=1}^{n}\left(\frac{G e_{i}(x)-\overline{G e}(x)}{\sigma(x)}\right)\left(\frac{G e_{i}(y)-\overline{G e}(y)}{\sigma(y)}\right)$.

(4) Network Density. $E$ is the set of network edges; the definition is shown in the following formula:

$$
\operatorname{den}(G)=\frac{2 \times|E|}{|V| \times(|V|-1)} .
$$

(5) Accuracy, Recall Rate, and F Measurement. F measures the accuracy of the neighborhood, recall and proximity values are mainly used to evaluate the degree of correspondence between the predicted functional unit and the actual functional unit, and its definition is shown in the following formula:

$$
N A(p, d)=\frac{\left|V_{P} \cap V_{b}\right|^{2}}{\left|V_{p}\right| \times\left|V_{b}\right|} .
$$

The following formulas, respectively, show the definition of the accuracy and recall rate of the module detection method:

$$
\begin{aligned}
\text { precision } & =\frac{N_{\mathrm{cp}}}{|P|}, \\
\text { recall } & =\frac{N_{\mathrm{cb}}}{|B|} .
\end{aligned}
$$

F-measure is the harmonic average of accuracy and response rate. It is used to measure the overall performance of the algorithm. The calculation method is given in the following formula:

$$
F-\text { measure }=\frac{2 \times \text { precision } \times \text { recall }}{\text { precision }+ \text { recall }} .
$$

(6) Sensitivity and Accuracy of Prediction. The definitions of the sensitivity and accuracy of prediction refer to the following formulas, respectively,

$$
\begin{array}{r}
\mathrm{Sn}=\frac{\sum_{i=1}^{n} \max _{j}\left\{T_{i j}\right\}}{\sum_{i-1}^{n} N_{i}}, \\
\mathrm{PPV}=\frac{\sum_{j=1}^{m} \max _{i}\left\{T_{i j}\right\}}{\sum_{j=1}^{m} T_{j}} .
\end{array}
$$

In the formula, $N_{\mathrm{i}}$ is the protein quality contained in the standard module $i$, and the $T_{j}=\sum_{i=1}^{n} T_{i j}$ accuracy is a complex assessment of the sensitivity and incidence of positive prognosis. Its definition is shown in the following formula:

$$
\text { Ace }=\sqrt{\mathrm{Sn} \times \mathrm{PPV}} .
$$

(7) $M M R$. In some cases, the standard function module can be compared with several prediction modules.

Measure the elephant and show its definition in following equation:

$$
\operatorname{MMR}(B, P)=\frac{\sum_{i=1}^{n} \max _{j=1}^{m} \mathrm{NA}\left(B_{i}, P_{i}\right)}{n} .
$$

When NA is a close value, the $\mathrm{B}$ and $P$ values are as described above, $n$ is the number of standard functional units, and $m$ is the number of prediction units.

(8) $P$ Value. $P$ value is a measurement technique that uses GO annotations to estimate the accuracy of predictors. It follows a hypergeometric distribution, and its definition is shown in the following formula:

$$
p-\text { value }=1-\sum_{i=0}^{k-1} \frac{\left(\begin{array}{c}
F \\
i
\end{array}\right)\left(\begin{array}{c}
N-F \\
n-i
\end{array}\right)}{\left(\begin{array}{c}
N \\
n
\end{array}\right)}
$$




\subsubsection{Multigranularity PPI Network Description Model}

(1) Functional Module Detection Algorithm Based on Multigranularity PPI Network Model and Ant Colony Optimization. The goal of the coarse-grained process is to reduce the size of the original PPI network and provide a smaller-scale robust coarse-grained network for the clustering algorithm.

The following equation shows the functional similarity of a pair of nodes $(i, j)$ :

$$
f_{i j}=\frac{\left|g^{i} \cap g^{j}\right|}{\left|g^{i} \cup g^{j}\right|} .
$$

In the formula, $g_{i}$ and $g_{i}$ represent the set of annotation elements of the GO function of $i$ and $j$, respectively.

Please refer to the network topology similarity as shown in

$$
d_{i j}=\frac{|\operatorname{Int}(i) \Delta \operatorname{Int}(j)|}{|\operatorname{Int}(i) \cup \operatorname{Int}(j)|+|\operatorname{Int}(i) \cap \operatorname{Int}(j)|} .
$$

The method of this problem searches for the shortest path, and the model of ant transition probability is given in the following formula:

$$
p_{i j}^{k}(t)= \begin{cases}\frac{\left[\tau_{i j}(t)\right]^{\alpha}\left[\eta_{i j}\right]^{\beta}}{\sum_{l \in U_{k}}\left[\tau_{i l}(t)\right]^{\alpha}\left[\eta_{i j}\right]^{\beta}}, & j \in U_{k}, \\ 0, & \text { otherwise. }\end{cases}
$$

The heuristic function is used in the NACO-FMD algorithm. The heuristic function uses GO information to improve the optimization goal. The specific calculation formula is listed in the following formula:

$$
\eta_{i j}=\frac{1}{d_{i j}}+f_{i j} .
$$

Therefore, the pheromone concentration remaining on each path must be continuously updated along with the optimization process. For information on the update method, see the following formulas:

$\tau_{i j}(t+n)=(1-p) \tau_{i j}(t)+\Delta \tau_{i j}(t)$,

$$
\Delta \tau_{i j}(t)=\sum_{k=1}^{m} \Delta \tau_{i j}^{k}(t),
$$

$$
\Delta \tau_{i j}^{k}(t)= \begin{cases}\frac{Q}{L_{k}}, & \text { The optimal pathofantKpassesthrough } a_{i j}, \\ 0, & \text { otherwise. }\end{cases}
$$

The formula for calculating modularity is given in

$$
D=\sum_{i=1}^{M} \frac{2 l_{i}-\bar{l}_{i}}{n_{i}}
$$

In the formula, $M$ is the number of modules, $l_{\mathrm{i}}$ is the number of interactions within a module, $\bar{l}_{i}$ is the number of interactions between modules, and $n_{\mathrm{i}}$ is the number of nodes in module $i$.

Table 2 shows the timing characteristics of the MGRACO-FMD algorithm and the NACO-FMD algorithm described using the hierarchical PPI network.

(2) Protein Functional Module Detection Framework Based on Multigranularity Model. The mechanism for determining the number of layers of particles $\mathrm{K}$ is determined by the following formula:

$$
K=\arg \min _{k}\left\{\left|\operatorname{den}\left(G_{k}\right)-\operatorname{den}\left(G_{k-1}\right)\right| \leq \mu, k>0\right\} .
$$

The determination of the modulus density is shown in

$$
\operatorname{den}\left(M_{l}\right)=\frac{2 \times\left|E_{l}\right|}{\left|V_{l}\right| \times\left(\left|V_{l}\right|-1\right)} .
$$

In order to fully test the efficiency of the structure, we will use 4 large PPI datasets, 3 of which are yeast datasets and the other is a human database. Table 3 provides detailed information on these 4 datasets.

Table 4 shows the influence of the parameter $\mu$ on the automatic determination of the number of particle layers and the execution time of the algorithm. Of course, the smaller the $\mu$, the more detailed the layer, and the shorter the running time of the algorithm.

Table 5 shows the number of different $\varepsilon_{1}$ levels of detail and the execution time of the algorithm.

\subsection{Data Mining}

3.2.1. The Basic Process of Data Mining. Figure 1 shows a schematic diagram of the data mining process.

3.2.2. Commonly Used Techniques for Data Mining. Many techniques are used in data mining, and the following techniques are commonly used:

(1) Forecasting Technology. Forecasts can be divided into macroforecasts and microforecasts [8]. Forecasting the economic development of a country or region is called macroforecasting, and forecasting the development prospects of a company is called microforecasting.

(2) Association Rules. Correlation refers to the existence of certain laws between data values, and the task of correlation analysis is to find the laws hidden between the data.

(3) Decision Tree. The decision tree model is a tree structure diagram. The highest point in the tree is called the root node, which simplifies a complex problem and improves efficiency [2].

3.2.3. Establishment of Computer Database for Data Mining. By using examples and rules to combine the design functions of the equipment, we can divide the process of the 
TABLE 2: The impact of PPI network multigranularity model on runtime.

\begin{tabular}{lcccc}
\hline \multirow{2}{*}{ Dataset } & \multicolumn{2}{c}{ MGRACO-FMD } & \multicolumn{2}{c}{ NACO-FMD } \\
& Optimization time $(\mathrm{s})$ & Total running time $(\mathrm{s})$ & Optimization time $(\mathrm{s})$ & Total running time $(\mathrm{s})$ \\
\hline MIPS & 966 & 1581 & 5821 & 4902 \\
DIP & 840 & 1203 & 9368 & 8777 \\
\hline
\end{tabular}

TABLe 3: Dataset in experiment with multigranularity structure.

\begin{tabular}{|c|c|c|c|c|c|c|}
\hline \multirow{2}{*}{$\begin{array}{l}\text { Datasets } \\
\text { DIPScere201 } \\
40703\end{array}$} & \multirow{2}{*}{$\begin{array}{c}\text { Http address } \\
\text { http://dip.doe-mbi.ucla.edu/dip/Do } \\
\text { wnIoad.cgi?SM }=\text { 7\&TX }=4932\end{array}$} & \multicolumn{2}{|c|}{$\begin{array}{c}\text { Source data size of } \\
\text { PI size }\end{array}$} & \multicolumn{3}{|c|}{ Preprocessed data layers size } \\
\hline & & 5126 & 22404 & 4 & 2803 & 14813 \\
\hline Mips & http://mips.helmhoItz-muenchen.de/proj/ppi/ & 4545 & 12318 & 4 & 2160 & 6683 \\
\hline BioGrid2014 & http://thebiogrid.org/download.php & 6334 & 80101 & 3 & 3817 & 40920 \\
\hline $\begin{array}{l}\text { DIPHsapi201 } \\
40703\end{array}$ & $\begin{array}{l}\text { http://dip.doe-mbi.ucla.edu/dip/Do } \\
\text { Wnload.cgi?SM = 7\&TX = 9606 }\end{array}$ & 4086 & 5823 & 3 & 2172 & 3264 \\
\hline
\end{tabular}

TABLE 4: The influence of different $\mu$ values on the number of particle layers and the running time of the algorithm.

\begin{tabular}{lccccc}
\hline \multirow{2}{*}{ Test item } & \multicolumn{5}{c}{$\mu$ values $\left(\times 10^{-4}\right)$} \\
& 0.5 & {$[1.5,3.5]$} & {$[4.5,6.5]$} & 7.5 & {$[8.5,9.5]$} \\
\hline Number of layers & 5 & 4 & 3 & 2 & 1 \\
Time & 793 & 710 & 810 & 992 & 1415 \\
\hline
\end{tabular}

TABLE 5: The effect of different $\varepsilon_{1}$ values on the number of levels of detail and the execution time of the algorithm.

\begin{tabular}{lcccccccc}
\hline \multirow{2}{*}{ Test item } & \multicolumn{10}{c}{$\varepsilon_{1}$ values } \\
& 0.1 & 0.2 & 0.3 & 0.4 & 0.5 & 0.6 & 0.7 & 0.8 \\
\hline Number of layers & 7 & 5 & 4 & 4 & 4 & 4 & 4 & 4 \\
Time & 583 & 466 & 615 & 668 & 710 & 740 & 759 & 770 \\
\hline
\end{tabular}

equipment design method into the following aspects based on the comprehensive consideration of the examples and rules:

(1) Preparation Stage. The main purpose of this stage is to obtain information about product clamping and process in order to prepare for the next design stage. In preparation for the improvement of subsequent procedures, the system has an interface for connecting to the CAPP system [3].

(2) Design Phase. The main function of this process is to retrieve the library of equipment chassis based on the clamping and process information received from the system, according to certain rules and algorithms, select the appropriate chassis from it, and obtain the desired design after the change.

\section{Application of Computer Artificial Intelligence System}

\subsection{Computer Artificial Intelligence Technology}

4.1.1. Computer Technology. Computer technology is a technical means used for computing, including hardware, software, and application technology. It is the cornerstone of artificial intelligence technology. As early as 1960, integrated circuit computers replaced transistor computers, greatly improving the computing power of computers [11]. Based on this, scientists have developed a statistical-based expert system technology that can perform calculations on large amounts of data and run probabilistic analysis. In computer technology, due to the lack of independent learning and analysis capabilities, this stage is more related to the calculation of mathematical logic, so it can be regarded as "pseudointelligence."

4.1.2. Artificial Intelligence Technology. The main task of artificial intelligence is to study theories, applications, and methods, so that human intelligence can be modeled and extended. This technical discipline studies the nature of intelligence and allows intelligent machines to respond to program instructions and make judgments on tasks to be processed similarly to human thinking. Artificial intelligence technology can be divided into three levels. The basic level is to combine data calculation and information storage so that machines can independently calculate and transmit information. The middle layer is where the machine can make decisions and actions based on judgments and decisions, and the last layer is where the machine automatically performs deep learning of algorithms and information sources to perform targeted actions [12]. At present, it has proven its capabilities in the military, medical, and Internet fields.

4.1.3. Artificial Intelligence Operating System. In the late 1960s, Stanford University's research on robot operating systems laid the theoretical foundation for artificial intelligence operating systems. Since then, with the gradual development of computer and network communication technologies, artificial intelligence operating systems have gradually matured and become more and more popular in military, aerospace, education, and home use. It is now generally believed that an artificial intelligence operating system should add one or more speech recognition systems, image processing systems, actuator systems, and cognitive 


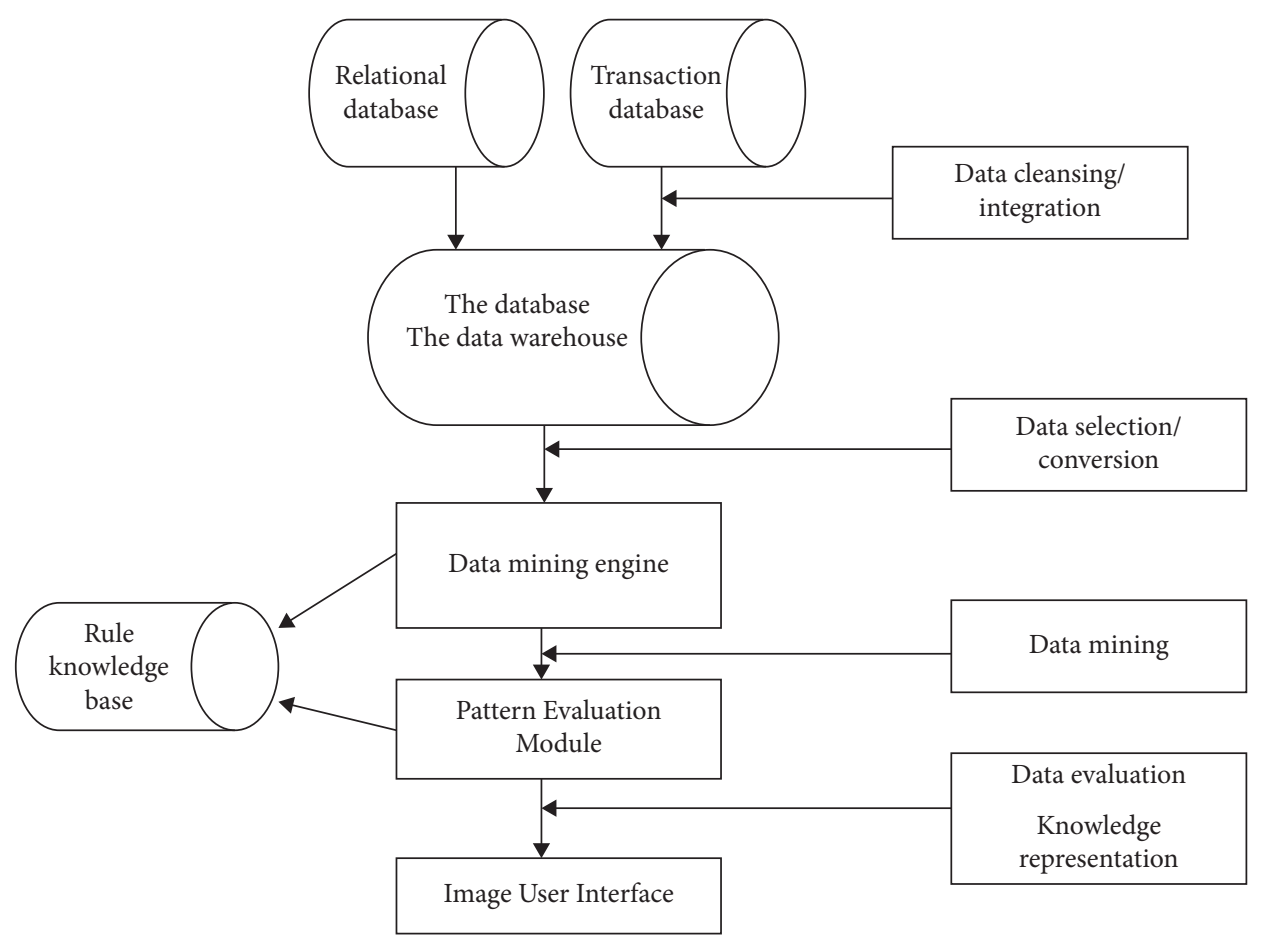

FIgURE 1: Basic data mining process.

behavior systems based on the core functions of a generalpurpose operating system [4]. Among them, the user can give instructions in the form of language through the voice recognition system and ask the machine to execute these instructions. The image processing system can recognize images and then perform tasks such as SLAM and navigation. The driving system can mainly use calculations such as motion algorithms. The logic is to perform functions such as gripper and chassis movement; the cognitive system enables the machine to have the ability of arguing and learning.

\subsection{Advantages of Computer Artificial Intelligence Systems}

4.2.1. Self-Learning Ability and Nonlinear Task Processing Ability. The main difference between artificial intelligence and traditional computer technology lies in their strong learning ability and nonlinear task processing ability. In the face of nonlinear problems, artificial intelligence first integrates and analyzes the low-level information that it contains and then obtains advanced concepts and information through information interpretation and information reasoning [1]. When the user operates the operating system, artificial intelligence can record and learn the user's habits and tendencies. Next time the same user uses them, they can actively view and filter the information, as shown in Figure 2.

4.2.2. Powerful Blur Processing and Collaboration Capabilities. The traditional operating mode of a computer is to issue various instructions through code source files and then perform various tasks. If the information graph is clear and the direction is clear, it can be

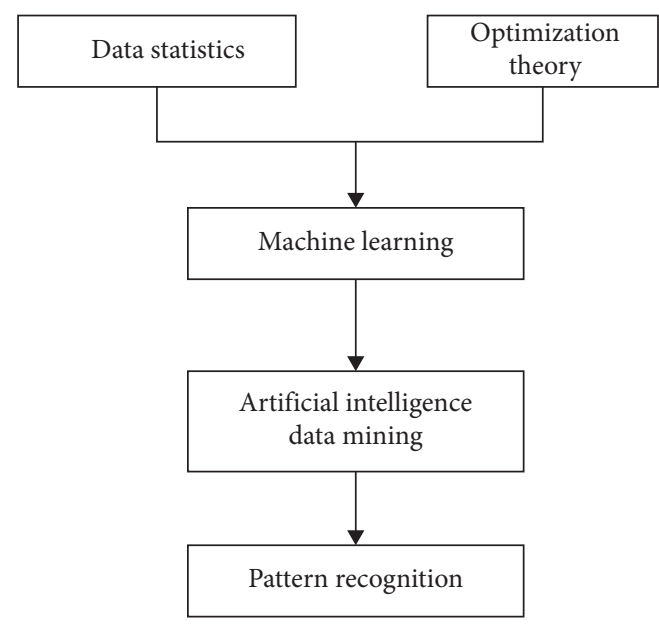

FIGURE 2: How artificial intelligence works.

processed effectively; if there is ambiguity, will be difficult to handle. Artificial intelligence imitates human wisdom and faces misinformation, which can promote correction through reasoning and self-correction. In addition, as the self-learning process increases, more similar information is processed, and the ability of artificial intelligence to process similar information gradually improves [24]. When traditional computing technology controls the Internet environment, query methods are usually used to introduce artificial intelligence into the management of the Internet environment, which can effectively avoid the problem of using the joint distributed idea of multiple agents to carry out the joint work of hierarchical technology management. 
4.2.3. Reduce Resource Consumption and Increase System Speed. When users use a computer, they usually run programs and give instructions. In this process, system redundancy often occurs. In severe cases, there may even be congestion or downtime. However, in addition to executing instructions given by people, artificial intelligence systems can also dynamically control the running system, close unnecessary programs in time, or solve accumulated problems, thereby freeing up computing resources and improving system efficiency. As China gradually enters the era of big data, the data that computers need to process will have the characteristics of multiple sources and structures [6]. However, traditional computer working models often lack processing capabilities when capturing large amounts of massive data. The problems are serious and often cause an error. After the introduction of artificial intelligence technology into the computer network system, these data with various attributes can be intelligently distributed to various levels to classify and control level by level, make more effective use of the logic processing capabilities of the machine, and improve system efficiency and maintain the system being stable.

\subsection{Application of Computer Artificial Intelligence System}

4.3.1. Application of Artificial Intelligence in Computer Software. In the past two decades, with the popularity of smart phones, the computer software field has undergone qualitative development, and considerable progress has been made in usability and performance. Excellent self-learning ability, ability of processing fuzzy information, and ability of collaborating with artificial intelligence technology have brought many new ideas to software development. For example, many information portals and online shops use expert systems. Use powerful artificial intelligence technology data mining to conduct personalized analysis of user habits, determine their click and purchase preferences, and better conduct accurate marketing [5]. The hardware terminals of Microsoft, Xiaomi, and other companies are equipped with intelligent voice assistants, so employees can give them instructions or perform simple voice-to-voice communication. At the same time, more and more companies are adopting telephone customer service software that includes intelligent voice recognition systems. Customers can use intelligent voice to solve more than $80 \%$ of the problems, and only the problems outside the existing intelligent voice database are called manual customer service. In this way, customers can use voice instead of regular keys to input basic information, thereby improving the user experience [25]. Customer service personnel do not have to answer repeated questions; they can devote their energy to creative work and better demonstrate their initiative. On the one hand, for companies, customer support services can be open 24/7 without restricting employees' working hours, which helps to improve the company's social image. On the other hand, maintaining or even improving the original service quality may also greatly affect the company's quality, operating expenses and profits. As practice increases, AI's autonomous learning capabilities can enable it to solve more problems, increase the size of the database, and better cope with the task at hand. In addition, with the gradual development of artificial intelligence image recognition systems, applications such as facial recognition technology, logistics robots, and autonomous driving technology have been developed, which has brought about further changes in people's work and lifestyle.

\subsubsection{Application of Artificial Intelligence in Computer} Equipment. Although the development of computer hardware is relatively slow compared with software, the combination of artificial intelligence and hardware technology still brings many important results. Among them, AI chips have become one of the most important development areas in the field of information technology [26]. The chip created by the deep learning function of artificial intelligence can not only realize independent learning and absorb new knowledge, but also shape the user's good personality according to the user's habits. However, in order to meet the computing needs of AI chips, matrix addition and multiplication are widely used to generate parallel processing of multiple processors [17]. As the calculation efficiency is effectively improved, the power consumption of the microcircuit is also increased, which leads to heat dissipation problems. Before there is an effective solution, the solution cannot be implemented in the actual production area. Integrate CPU, DIC, etc. In the way of integration and interconnection, thus create a good environment foundation for software work. In addition, the use of artificial intelligence helps to establish effective communication between the entire computer and external equipment, thereby contributing to the scientific and rationality of the equipment structure.

\subsubsection{Apply Artificial Intelligence to Big Data Management.} Compared with traditional computer technology, it has the unique ability of processing fuzzy information and the ability of using artificial intelligence for autonomous learning. It has obvious advantages in data collection and mining. In view of the massive amount of data on the Internet, this not only makes the system overwhelmed and collects all target information indiscriminately, but also increases resource consumption and may extract too much inappropriate information, which greatly discounts the value of data collection. Artificial intelligence can obtain the most accurate and relevant data in a relatively short period of time, so as to classify and research user preferences.

\subsubsection{Application of Artificial Intelligence in Local Computer} Security Management. Compared with traditional firewalls, the accuracy and efficiency of smart firewalls play an important role in virus detection and surveillance [19]. Using intelligent analysis and identification functions, suspicious objects can be compared with existing malicious information to determine the most appropriate deletion method and control their access rights. In the face of hacker attacks, virus attacks, and other risks, you can also wisely disable certain 
ports or local networks to effectively control the spread of viruses and improve the security of your local computer. In addition, the intelligent firewall software can also carry out advanced control of the application software on the computer, grant it system privileges, and solve the problem of denial of service.

4.3.5. Use Artificial Intelligence When Planning Computer Networks and Making Decisions. The use of artificial intelligence systems to manage the computer network can be combined with knowledge databases and problem-solving techniques to control the computer network in a more professional way. For example, an AI antispam application can block spam that may endanger user privacy in time and automatically classify and mark received emails, thereby increasing the utilization of internal mailboxes. The powerful logic calculation function of the artificial intelligence system can understand how users process information and then optimize their reasoning and judgment skills. Combined with data mining, they can identify and delete redundant content on computer networks, solve instability problems, and improve computational efficiency through intelligent network planning.

\section{Conclusion}

The manufacturing industry plays a key role in ensuring national security, national defense, high technology, and creating living standards and prosperity. With the acceleration of global economic integration, the manufacturing industry must improve its competitiveness in the global market and then adapt its products to the region and personal requirements to best meet the diverse needs of customers. The key to solving this problem lies in the development of technology, which can improve the efficiency of mass production of nonstandard, small, and mediumsized products. The emergence of computer-aided design of equipment makes this technology possible.

Using calculation methods to effectively identify PPI network functional modules has become an important research field in the field of modern bioinformatics. In recent years, many calculation methods with higher recognition accuracy have appeared, but many of them have higher time complexity. With limited computing resources, these algorithms cannot meet the requirement of analyzing largescale PPI networks. At the same time, with the development of computer technology, artificial intelligence appeared, and the use of artificial intelligence systems in computer technology can contribute to the further development of artificial intelligence. In addition, in the data mining process, the artificial intelligence system can better respond to calls and extract different levels of content from information with different characteristics and combine the functions of thinking and judgment to aggregate and refine high-quality information, thereby improving data efficiency of analysis. Compared with traditional computer technology, the unique characteristics of artificial intelligence systems help to update computer software and hardware technology and maintain the security and stability of computer networks and improve operating efficiency in accordance with the requirements of the new era.

\section{Data Availability}

The datasets used and/or analyzed during the current study are available from the corresponding author on reasonable request.

\section{Conflicts of Interest}

The authors declare no conflicts of interest.

\section{References}

[1] Y. Xie, W. Zhang, C. Li, S. Lin, Y. Qu, and Y. Zhang, "Discriminative object tracking via sparse representation and online dictionary learning," IEEE Transactions on Cybernetics, vol. 44, no. 4, pp. 539-553, 2014.

[2] D. Comaniciu, V. Ramesh, and P. Meer, "Kernel-based object tracking," IEEE Transactions on Pattern Analysis and Machine Intelligence, vol. 25, no. 5, pp. 564-577, 2003.

[3] S. S. Dhanda, B. Singh, and P. Jindal, "Lightweight cryptography: a solution to secure IoT," Wireless Personal Communications, vol. 112, pp. 1-34, 2020.

[4] H. Grabner, M. Grabner, and H. Bischof, "Real-time tracking via on-line boosting," in Proceedings of British Machine Vision Conference, pp. 47-56, Edinburgh, UK, September 2006.

[5] L. Shijian and C. L. Tan, "Script and language identification in noisy and degraded document images," IEEE Transactions on Pattern Analysis and Machine Intelligence, vol. 30, pp. 14-24, 2007.

[6] J. N. Luo, M. L. Li, X. Z. Liu, W. H. Tian, S. M. Zhong, and K. B. Shi, "Stabilization analysis for fuzzy systems with a switched sampled-data control," Journal of the Franklin Institute, vol. 357, no. 1, pp. 39-58, 2020.

[7] G. Lentka and S. Tyborczyk, "Distributed measurement system with data transmission secured using XXTEA algorithm," Przeglad Elektrotechniczny, vol. 92, no. 10, pp. 266-269, 2016.

[8] G. Lian, "A novel real-time object tracking based on kernelized correlation filter with self-adaptive scale computation in combination with color attribution," Journal of Ambient Intelligence and Humanized Computing, 2020.

[9] X. Xue Mei and H. Haibin Ling, "Robust visual tracking and vehicle classification via sparse representation," IEEE Transactions on Pattern Analysis and Machine Intelligence, vol. 33, no. 11, pp. 2259-2272, 2011.

[10] J. Ning, J. Yang, S. Jiang, L. Zhang, and M. Yang, "Visual tracking via dual linear structured SVM and explicit feature map," in Proceedings of the IEEE Conference on Computer Vision and Pattern Recognition(CVPR), pp. 4266-4274, Las Vegas, NV, USA, June 2016.

[11] E. Punarselvam, S. Mohamed Yacin, B. Mohsen, N. B. Prakash, T. Jayasankar, and S. Sudhakar, "Different Loading Condition and Angle Measurement of Human Lumbar Spine MRI Image Using ANSYS," Journal of Ambient Intelligence and Humanized Computing, vol. 12, p. 11, 2020.

[12] P. Sahare, R. E. Chaudhari, and S. B. Dhok, "Word level multiscript identification using curvelet transform in log-polar domain," IETE Journal of Research, vol. 65, no. 3, pp. 410-432, 2019. 
[13] Y. Lin, H. Song, F. Ke, W. Yan, Z. Liu, and F. Cai, "Optimal caching scheme in D2D networks with multiple robot helpers," Computer Communications, vol. 181, pp. 132-142, 2022.

[14] X. Wu, W. Zheng, X. Chen, Y. Zhao, T. Yu, and D. Mu, "Improving high-impact bug report prediction with combination of interactive machine learning and active learning," Information and Software Technology, vol. 133, Article ID 106530, 2021.

[15] Y. Jiang and X. Li, "Broadband cancellation method in an adaptive co-site interference cancellation system," International Journal of Electronics, 2021.

[16] B. Babenko, M. Ming-Hsuan Yang, and S. Belongie, "Robust object tracking with online multiple instance learning," IEEE Transactions on Pattern Analysis and Machine Intelligence, vol. 33, no. 8, pp. 1619-1632, 2011.

[17] P. Shivakumara, Z. Yuan, D. Zhao, T. Lu, and C. L. Tan, "New gradient-spatial-structural features for video script identification," Computer Vision and Image Understanding, vol. 130, pp. 35-53, 2015.

[18] H. Grabner, C. Leistner, and H. Bischof, "Semi-supervised online boosting for robust tracking," Lecture Notes in Computer Science, in Proceedings of European Conference on Computer Vision, pp. 234-247, Marseille, France, October 2008.

[19] D. Wang, H. Lu, and M.-H. Yang, "Robust visual tracking via least soft-threshold squares," IEEE Transactions on Circuits and Systems for Video Technology, vol. 26, no. 9, pp. 17091721, 2016.

[20] J. Yu, L. Lu, Y. Chen, Y. Zhu, and L. Kong, "An indirect eavesdropping attack of keystrokes on touch screen through acoustic sensing," IEEE Transactions on Mobile Computing, vol. 20, no. 2, pp. 337-351, 2021.

[21] S. Zhao, F. Li, H. Li et al., "Smart and practical privacypreserving data aggregation for fog-based smart grids," IEEE Transactions on Information Forensics and Security, vol. 16, pp. 521-536, 2021.

[22] S. Hare, S. Golodetz, A. Saffari et al., "Struck: structured output tracking with kernels," IEEE Transactions on Pattern Analysis and Machine Intelligence, vol. 38, no. 10, pp. 20962109, 2016.

[23] A. Amiruddin, A. A. P. Ratna, and R. F. Sari, "Construction and analysis of key generation algorithms based on modified fibonacci and scrambling factors for privacy preservation," IJ Netw Secur, vol. 21, no. 2, pp. 250-258, 2019.

[24] R. Yao, Q. Shi, C. Shen, Y. Zhang, and A. V. D. Hengel, "Partbased visual tracking with online latent structural learning," in Proceedings of IEEE Conference on Computer Vision and Pattern Recognition (CVPR), pp. 2363-2370, Portland, OR, USA, June 2013.

[25] J. Zhang, S. Ma, and S. Sclaroff, "MEEM: robust tracking via multiple experts using entropy minimization," in Proceedings of European Conference on Computer Vision, pp. 188-203, Zurich, Switzerland, September 2014.

[26] K. Zhang and H. Song, "Real-time visual tracking via online weighted multiple instance learning," Pattern Recognition, vol. 46, no. 1, pp. 397-411, 2013. 